\title{
New Consensus on the Management of Barrett's Dysplasia and Early Stage Esophageal Adenocarcinoma: Limited Evidence, but Best Available Guidance
}

(Gastroenterology 2012;143:336-346)

Yunju Jo

Division of Gastroenterology, Department of Internal Medicine, Eulji University School of Medicine, Eulji General Hospital, Seoul, Korea

\section{Summary}

Barrett's esophagus (BE) is important as a precursor lesion of esophageal adenocarcinoma (EA), which has recently been rapidly increasing in incidence in the Western world. The absolute risk in individual patients with reflux esophagitis is less than 1 in 1000 per annum. ${ }^{1}$ In the United States, the annual incidence of EA in Caucasian men is 3.6/100,000 compared to 0.8 in African American men and 0.3 in Caucasian women. ${ }^{2}$ The incidence of EA will be expected to increase as the incidence of gastroesophageal reflux disease increases in the Republic of Korea.

The guideline for diagnosis, surveillance, and therapy of $\mathrm{BE}$ was published by the American College of Gastroenterology in 1998 and updated in 2008. ${ }^{3}$ The guideline from the United Kingdom has also been updated on the Web. ${ }^{1}$ Notably, consensus on management of Barrett's dysplasia and early-stage EA has recently been reached, ${ }^{4}$ and it is thus introduced here.

These guidelines were developed by international, multi- disciplinary experts through systematic and evidence-based reviews of different strategies for treating patients with $\mathrm{BE}$ and dysplasia or early stage EA. Consensus statements were developed by the Delphi process. Until consensus for each statement reached above $80 \%$ agreement, there were total of 4 rounds of repeated anonymous voting. As a result, 81 of the 91 statements achieved consensus, including the following 8 clinical statements: (1) specimens from endoscopic resection (ER) are better than biopsies for staging lesions, (2) it is important to carefully map the size of the dysplastic areas, (3) patients that receive ablative or surgical therapy require endoscopic follow-up, (4) high-resolution endoscopy is necessary for accurate diagnosis, (5) endoscopic therapy for high grade dysplasia (HGD) is preferred to surveillance, (6) endoscopic therapy for HGD is preferred to surgery, (7) the combination of endoscopic resection and radiofrequency ablation is the most effective therapy and (8) after endoscopic removal of lesions from patients with $\mathrm{HGD}$, all areas of $\mathrm{BE}$ should be ablated.

Received: September 15, 2012 Revised: September 18, 2012 Accepted: September 19, 2012

(c) This is an Open Access article distributed under the terms of the Creative Commons Attribution Non-Commercial License (http://creativecommons. org/licenses/by-nc/3.0) which permits unrestricted non-commercial use, distribution, and reproduction in any medium, provided the original work is properly cited.

*Correspondence: Yunju Jo, MD, PhD

Division of Gastroenterology, Department of Internal Medicine, Eulji University School of Medicine, Eulji General Hospital, Hangeulbiseong-ro 68, Nowon-gu, Seoul 139-711, Korea

Tel: +82-2-970-8209, Fax: +82-2-970-8621, E-mail: jyj1138@eulji.ac.kr

Financial support: None.

Conflicts of interest: None. 


\section{Comment}

This consensus guidance focused on statements concerning HGD and EA rather than lower grade dysplasia. In addition, it has new informative statements updated from the previous guideline for BE. I would introduce some of them with comments.

(1) In terms of diagnosis of BE and HGD, it was suggested that at least 2 experienced gastrointestinal pathologists should evaluate all Barrett's biopsies because of poor inter-observer agreement in distinguishing HGD from intra-mucosal adenocarcinoma. ${ }^{5}$ However, we wonder about several complicated problems such as insurance, cost-effectiveness, or agreement of the pathologists during clinical application of this guidance.

(2) Non-goblet columnar metaplasia of the esophagus can progress to cancer. The incidence of cancer progression from BE was similar in patients with intestinal metaplasia and without intestinal metaplasia. ${ }^{6}$ The degree of malignant potential in nongoblet columnar metaplasia is a contentious issue, but it has led to changes in the definition of $\mathrm{BE}$ at this time. However, the risk of malignant progression among patients with $\mathrm{BE}$ was comparatively lower than previous expectations, ${ }^{7}$ so that currently recommended surveillance strategies may not be cost-effective.

(3) The recommended method of surveillance for patients with $\mathrm{BE}$ and with HGD was high-resolution endoscopes and 4-quadrant targeted biopsies at 1-2 cm intervals. Although scientific evidence for this recommendation was very low, standard-resolution endoscope was not recommended. In terms of surveillance or management for Barrett's dysplasia and EA, I wonder how effective it is in clinical setting, because this recommendation requires referral only to a tertiary hospital.

(4) With regard to management of HGD and esophageal mucosal cancer, ER is recommended as a diagnostic and staging tool, and endoscopic therapy is preferred over esophagectomy. According to the previous guideline, ${ }^{3}$ the recommended approach for management of patients with HGD was dependent on local expertise, both endoscopic and surgical, along with the patient's age, co-mobidity, and preference. Pech et al $^{8}$ recently published a retrospective cohort study on either ER or esophagectomy for Barrett's-associated intramucosal carcinoma, and concluded that both esophagectomy and ER are effective treatment modalities. However, clinicians should explain that the recurrence rate is higher in patients treated with ER, while surgery is associated with higher morbidity and mortality. In addition, clinicians should assess the capacity of hospital to handle such cases, specifically whether the institution has the necessary endoscopic equipment and expert endoscopists, or whether it is a high-volume center for esophagectomy.

These consensus statements have some limitations: the overall quality of evidence related to the statements was low, and some geographical areas were under-represented in the data. The Barrett's esophagus group conducted 4 times of voting, with a priori threshold for consensus defined as $\geq 80 \%$ strongly agreeing or agreeing with reservation to a statement. Through the process of repeated voting, the experts were persuaded to change their mind to reach consensus, overcoming imperfect evidence.

Though there remains heterogeneity in the management of HGD and early EA throughout the world, this consensus on treating BE patients with dysplasia and early EA will help the practicing clinician make the best possible treatment decisions given the limited research data.

\section{References}

1. Guidelines for the diagnosis and management of Barrett's columnar-lined oesophagus. A report of the working party of the British Society of Gastroenterology 2005. Available from URL: http://www. bsg.org.uk/images/stories/docs/clinical/guidelines/oesophageal/ Barretts_Oes.pdf (accessed September 2012).

2. Brown LM, Devesa SS. Epidemiologic trends in esophageal and gastric cancer in the United States. Surg Oncol Clin N Am 2002;11: 235-256.

3. Wang KK, Sampliner RE; Practice Parameters Committee of the American College of Gastroenterology. Updated guidelines 2008 for the diagnosis, surveillance and therapy of Barrett's esophagus. Am J Gastroenterol 2008;103:788-797.

4. Bennett C, Vakil N, Bergman J, et al. Consensus statements for management of Barrett's dysplasia and early-stage esophageal adenocarcinoma, based on a Delphi process. Gastroenterology 2012;143:336346.

5. Ormsby AH, Petras RE, Henricks WH, et al. Observer variation in the diagnosis of superficial oesophageal adenocarcinoma. Gut 2002; 51:671-676.

6. Gatenby PA, Ramus JR, Caygill CP, Shepherd NA, Watson A Relevance of the detection of intestinal metaplasia in non-dysplastic columnar lined oesophagus. Scand J Gastroenterol 2008;43:524530.

7. Bhat $\mathrm{S}$, Coleman HG, Yousef $\mathrm{F}$, et al. Risk of malignant progression in Barrett's esophagus patients: results from a large population-based study. J Natl Cancer Inst 2011;103:1049-1057.

8. Pech O, Bollschweiler E, Manner H, Leers J, Ell C, Hölscher AH. Comparison between endoscopic and surgical resection of mucosal esophageal adenocarcinoma in Barrett's esophagus at two high-volume centers. Ann Surg 2011;254:67-72. 